\title{
ON THE NONEXISTENCE OF ELEMENTS OF HOPF INVARIANT ONE
}

\author{
BY J. F. ADAMS \\ Communicated by S. Eilenberg, April 29, 1958
}

With the usual definitions of homotopy-theory, we have the following theorem.

THEOREM 1. (a) $S^{n-1}$ is not an $H$-space unless $n=2,4$, or 8 .

(b) There is no element of Hopf invariant one in $\pi_{2 n-1}\left(S^{n}\right)$ unless $n=2,4$, or 8 .

For the context of this question, see [5] (especially pp. 436-438), $[4$, Chapter VI] and $[6, \S \S 20,21]$.

This theorem results from reasonings with secondary cohomology operations. It is generally understood that a secondary operation corresponds to a relation between primary operations. One may formalize the notion of a "relation" by introducing pairs $(d, z)$, algebraic in nature, as follows.

Let $p$ be a prime; let $A$ be the Steenrod algebra [2, p. 43] over $Z_{p}$. One defines the notion of a graded left module $M$ over the graded algebra $A$ so that $M=\sum_{q} M_{q}$ and $A_{q} M_{r} \subset M_{q+r}$. For example, let us write $H^{q}(X)$ for $H^{q}\left(X ; Z_{p}\right), H^{*}(X)$ for $\sum_{q} H^{q}\left(X ; Z_{p}\right)$ and $H^{+}(X)$ for $\sum_{q>0} H^{q}\left(X ; Z_{p}\right)$; then $H^{*}(X)$ and $H^{+}(X)$ are graded left modules over $A$. Let $M, N$ be such modules; one defines the notion of an $A$-map $f: M \rightarrow N$ of degree $r$ so that $f\left(M_{q}\right) \subset N_{q+r}$.

A pair $(d, z)$, then, is to have the following nature. The first entry $d$ is to be an $A$-map $d: C_{1} \rightarrow C_{0}$ of degree zero. Here $C_{0}, C_{1}$ are to be modules in the above sense; we require, moreover, that they are locally finitely-generated and free, and that $\left(C_{i}\right)_{q}=0$ if $q<i(i=0,1)$. The second entry $z$ is to be a homogeneous element of Ker $d$.

Let $(d, z)$, then, be a pair of this sort. We call $\Phi$ a stable secondary cohomology operation associated with $(d, z)$, if it satisfies the following axioms.

Axiom (1). $\Phi(\epsilon)$ is defined for each $A$-map $\epsilon: C_{0} \rightarrow H^{+}(X)$ of degree $m \geqq 1$ and such that $\epsilon d=0$.

Such a map $\epsilon$ is determined by its values on the elements of an $A$-base of $C_{0}$. It therefore corresponds to a set of elements of $H^{+}(X)$. In particular, if $C_{0}$ is free on one given generator $c$, we write $u=\epsilon c$; we may thus consider $\Phi$ as a function of one variable $u$, where $u$ runs over a subset of $H^{+}(X)$. In this case we write $\Phi(u)$ for $\Phi(\epsilon)$.

For the next axiom, set $\operatorname{deg}(z)=n+1$, let $f: C_{1} \rightarrow H^{+}(X)$ run over 
the $A$-maps of degree $(m-1)$, and let $Q^{m+n}(d, z ; X)$ be the set of elements of the form $f z$.

Axiom (2). $\Phi(\epsilon) \in H^{m+n}(X) / Q^{m+n}(d, z ; X)$.

For the next axiom, let $g: Y \rightarrow X$ be a map.

AxIOM (3). $g^{*} \Phi(\epsilon)=\Phi\left(g^{*} \epsilon\right)$.

For the next axiom, let $(X, Y)$ be a pair, and let $\epsilon: C_{0} \rightarrow H^{+}(X)$ be a map of degree $m \geqq 1$ such that $\epsilon d=0$ and $i^{*} \epsilon=0$. We can now form the following diagram.

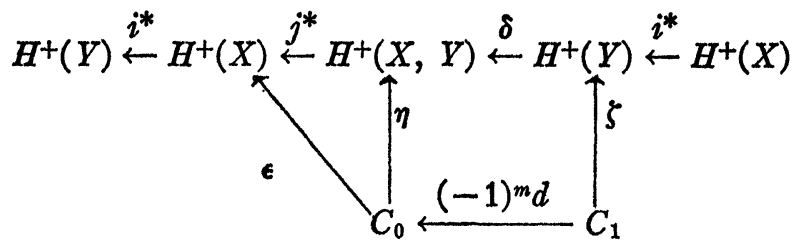

Axiom (4). $i^{*} \Phi(\epsilon)=\{\zeta z\} \bmod i^{*} Q^{m+n}(d, z ; X)$.

For the next axiom, let $S X$ be the suspension of $X$, and let $\sigma: H^{+}(X) \rightarrow H^{+}(S X)$ be the suspension isomorphism. Let $\epsilon$ be as above.

Axiom (5). $\sigma \Phi(\epsilon)=\Phi(\sigma \epsilon)$.

THEOREM 2. Given any pair $(d, z)$ (as above), there is at least one stable secondary cohomology operation $\Phi$ associated with it (in the sense of the axioms above).

This theorem is proved by the method of the universal example. The next theorem allows us to study the operations $\Phi$ by applying homological algebra (see $[3])$ to the pairs $(d, z)$.

THEOREM 3. (a) If $\Phi, \Phi^{\prime}$ are two operations associated with the same pair $(d, z)$ then there is an element $c$ in $\left(C_{0} / d C_{1}\right)_{n}$ such that

$$
\Phi(\epsilon)-\Phi^{\prime}(\epsilon)=\{\epsilon c\} .
$$

(b) Suppose given $d$ (as above), elements $z_{t}$ in Ker $d$, and operations $\Phi_{t}$ associated with the pairs $\left(d, z_{t}\right)$. Suppose $z=\sum_{t} a_{t} z_{t}\left(a_{t} \in A\right)$. Then there is an operation $\Phi$ associated with $(d, z)$ such that

$$
\sum_{t} a_{t} \Phi_{t}(\epsilon)=\{\Phi(\epsilon)\} \bmod \sum_{t} a_{t} Q^{m+n t}\left(d, z_{t} ; X\right) .
$$

(c) Suppose given a diagram

$$
\begin{gathered}
C_{1} \stackrel{m_{1}}{\longrightarrow} C_{1}^{\prime} \\
d \downarrow \stackrel{m_{0}}{\longrightarrow} C_{0}^{\prime} \\
C_{0} \stackrel{d^{\prime}}{ }
\end{gathered}
$$


in which $d, d^{\prime}$ are as above, and $m_{0}, m_{1}$ are $A$-maps of degree zero. Let $\Phi$ be an operation associated with a pair $(d, z)$. Then there is an operation $\Phi^{\prime}$ associated with $\left(d^{\prime}, m_{1} z\right)$ such that

$$
\Phi\left(\epsilon^{\prime} m_{0}\right)=\left\{\Phi^{\prime}\left(\epsilon^{\prime}\right)\right\}
$$

for each $\epsilon^{\prime}: C_{0}^{\prime} \rightarrow H^{+}(X)$ of the sort considered above.

One may show that for operations in one variable, there is a Cartan formula for expanding $\Phi(u v)$, where $u v$ is a cup-product.

We now take $p=2$. We also omit to summarize some work with homological algebra. This work leads us to consider certain pairs $(d, z)$. By applying Theorem 2 , we obtain secondary operations $\Phi_{i, j}(u)$ for $0 \leqq i \leqq j, j \neq i+1$. The operation $\Phi_{j, i}(u)$ is of degree $2^{i}+2^{j}-1$, and it is defined on classes $u$ such that $S q^{2^{r}}(u)=0$ for $0 \leqq r \leqq j$.

Let $P$ be complex projective space of infinitely-many dimensions, and let $y$ be a generator of $H^{2}(P)$ (by which we mean $H^{2}\left(P ; Z_{2}\right)$ ). We may ask for the values of the operations $\Phi_{i, j}$ in $H^{*}(P)$. Now, $\Phi_{i, j}\left(y^{r}\right)$ is defined only if $r \equiv 0$ and $\bmod 2^{j}$. Moreover, the degree of $\Phi_{i, j}$ is odd unless $i=0$ and $j>0$; so that $\Phi_{i, j}\left(y^{r}\right)$ lies in a zero group unless $i=0$ and $j>0$. It remains only to consider $\Phi_{0, j}\left(y^{n 2^{j}}\right)$; this is defined modulo zero.

THEOREM 4.

$$
\Phi_{0, j}\left(y^{n 2^{j}}\right)=n y^{(n+1 / 2) 2^{j}}
$$

(mod zero).

In the proof of this theorem we make essential use of a formula for the composite operation $\Phi_{0, j} S q^{2^{j}}$. This formula is proved by applying Theorem 3.

THEOREM 5. For each $k \geqq 3$ we have a formula

$$
\sum_{i, j ; j \leqq k} a_{i, j, k} \Phi_{i, j}(u)=S q^{2^{k+1}}(u) \quad(\bmod Q) .
$$

The formula is valid on classes $u$ such that $S q^{2^{r}}(u)=0$ for $0 \leqq r \leqq k$, and holds modulo a certain subgroup $Q$. It is proved as follows. By applying Theorem 3 , we obtain a formula

$$
\sum_{i, j ; j \leqslant k} a_{i, j, k} \Phi_{i, j}(u)=\lambda S q^{2^{k+1}}(u)
$$

in which $a_{i, j, k} \in A$, and the coefficient $\lambda$ remains to be determined. Applying the formula to a suitable class $u$ in $H^{*}(P)$, we determine $\lambda=1$.

To prove Theorem 1 , it is sufficient to prove it for the case $n=2^{m}$. This case follows immediately from Theorem 5, using the same argument as that used by Adem $[1, \S 4]$ in the case $n \neq 2^{m}$. 


\section{REFERENCES}

1. J. Adem, The iteration of the Steenrod squares in algebraic topology, Proc. Nat. Acad. Sci. U.S.A. vol. 38 (1952) pp. 720-726.

2. H. Cartan, Sur l'itération des opérations de Steenrod, Comment. Math. Helv. vol. 29 (1955) pp. 40-58.

3. H. Cartan and S. Eilenberg, Homological algebra, Princeton University Press, 1956.

4. P. J. Hilton, An introduction to homotopy theory, Cambridge University Press, 1953.

5. H. Hopf, Über die Abbildungen von Sphären auf Sphären niedriger Dimension, Fund. Math. vol. 25 (1935) pp. 427-440.

6. N. E. Steenrod, The topology of fibre bundles, Princeton University Press, 1951.

INSTITUTE FOR ADVANCED STUDY 